\title{
The Origin of Separable States and Separability Criteria from Entanglement-breaking Channels
}

\author{
Banghai Wang ${ }^{1,2}$, Qin Li $^{1}$ and Dongyang Long ${ }^{1}$ \\ ${ }^{1}$ Department of Computer Science, Sun Yat-sen University, Guangzhou 510006, \\ P.R.China \\ ${ }^{2}$ Faculty of Computer, Guangdong University of technology, Guangzhou 510006, \\ P.R.China \\ E-mail: wangbanghai@gmail.com, liqin805@163.com, issldy@mail.sysu.edu.cn
}

\begin{abstract}
In this paper, we show that an arbitrary separable state can be the output of a certain entanglement-breaking channel corresponding exactly to the input of a maximally entangled state. A necessary and sufficient separability criterion and some sufficient separability criteria from entanglement-breaking channels are given.

PACS numbers: 03.67.-a, 03.65.Ta
\end{abstract}


The Origin of Separable States and Separability Criteria from Entanglement-breaking Channels2

\section{Introduction}

Quantum entanglement, which is applied to various types of quantum information processing such as quantum computation[1], quantum dense coding[2], quantum teleportation [3, quantum cryptography [4], etc., lies in the heart of quantum information theory. However, quantum entanglement is not yet fully understood by people, and many problems on entanglement remain open [5]. It is one of the central problems to check whether or not a state is entangled. Some necessary 6, 7, 8, 9, 10, 11, 12, 13, or sufficient [14, 15] separability criteria, as well as the necessary and sufficient separability criterion for low dimension states [16], have been found. Unfortunately, there has not been any effective necessary and sufficient separable condition for an arbitrary state yet.

Entanglement underlines the intrinsic order of statistical relations between subsystems of a composite quantum system [5, 17]. In this paper, we show that there exists a correlation between the separable state and a maximally entangled state in any quantum systems of arbitrary dimensions from the entanglement-breaking channel (EBC). This paper is organized as follows. Sec. II demonstrates that all separable states can be the output of certain EBCs corresponding exactly to the input of a maximally entangled state. The separability criteria from EBCs are investigated in Sec. III. Sec. IV summarizes our main results.

\section{The origin of all separable states can be a maximally entangled state}

Let $\mathcal{H}_{A}, \mathcal{H}_{B}$ be two Hilbert spaces, $\mathcal{H}_{A, B}$ be the tensor product space $\mathcal{H}_{A} \otimes \mathcal{H}_{B}$. Denote $\mathcal{B}\left(\mathcal{H}_{A, B}\right)$ as the set of operators on $\mathcal{H}_{A, B}, \mathcal{B}\left(\mathcal{H}_{A, B}\right)^{+}$as the subset of positive semidefinite operators (i.e. the unnormalized density operators) on $\mathcal{H}_{A, B}$. Let $\operatorname{dim} \mathcal{H}_{A}=d_{A}$ and $\operatorname{dim} \mathcal{H}_{B}=d_{B}$. A mixed state $\rho \in \mathcal{B}\left(\mathcal{H}_{A, B}\right)^{+}$is called separable state if it can be written as

$$
\rho=\sum_{k} p_{k}\left|\psi_{k}\right\rangle\left\langle\psi_{k}|\otimes| \phi_{k}\right\rangle\left\langle\phi_{k}\right|
$$

where $\left\{p_{k}\right\}$ is a probability distribution, $\left|\psi_{k}\right\rangle$ and $\left|\phi_{k}\right\rangle$ are pure states of $\mathcal{H}_{A}$ and $\mathcal{H}_{B}$ respectively.

A quantum channel is a completely positive (CP) linear map [18, 19]. The EBC is the class of quantum channels, for which $(I \otimes \Phi)(\rho)$ is always separable for any state $\rho$, where $\rho$ is separable or not [20, 21, 22]. By Ref. [22], an EBC can be written in either of the following equivalent forms

$$
\begin{aligned}
\Phi(\sigma) & =\sum_{k} R_{k} \operatorname{Tr} F_{k} \sigma \\
& =\sum_{k}\left|\psi_{k}\right\rangle\left\langle\psi_{k}\right|\left\langle\phi_{k}|\sigma| \phi_{k}\right\rangle,
\end{aligned}
$$

where $\sigma$ is any density operator, $R_{k}$ is a density operator and $F_{k}$ is a positive semi-definite operator. The channel $\Phi$ is entanglement-breaking and trace-preserving(EBT) if and only if $\sum_{k} F_{k}=\sum_{k}\left|\phi_{k}\right\rangle\left\langle\phi_{k}\right|=I$, where the set $\left\{F_{k}\right\}$ form a POVM. The properties of 
EBT were investigated by many people[20, 21, 22, 23]. The action of an EBT can be substituted by a measurement and state-preparation protocol[24]: The sender makes a measurement on the input state $\sigma$ by means of a POVM $\left\{F_{k}\right\}$, and sends the outcome $k$ via a classical channel to the receiver. Then the receiver prepares an agreed-upon state $R_{k}[21,25]$.

However, a general channel need be not trace-preserving, and a channel is tracepreserving if no loss of the particle[18, 19]. Any finite-dimensional completely positive trace-preserving (CPT) linear map $\Phi$ can be represented as

$$
\Phi(\sigma)=\sum_{k} E_{k} \sigma E_{k}^{\dagger}
$$

where the $E_{k}$ are complex matrices satisfying

$$
\sum_{k} E_{k} E_{k}^{\dagger}=I
$$

In this paper, we consider

$$
\sum_{k} E_{k} E_{k}^{\dagger} \leq I
$$

i.e. the channel has only the completely positive property and need not trace-preserving. Some properties hold for the channel without trace-preserving but not for the channel with trace-preserving, vice verse.

We need two easily proved lemmas.

Lemma 1. 26] A pure bipartite state $|\beta\rangle \in \mathbb{C}^{d_{A}} \otimes \mathbb{C}^{d_{B}}\left(d_{B} \geq d_{A}\right)$ is a maximally entangled if and only if $E(|\beta\rangle)=\log _{2} d_{A}$, where $E(|\beta\rangle)=-\operatorname{Tr}\left(\rho_{A(B)} \log _{2} \rho_{A(B)}\right)$ and $\rho_{A(B)}=\operatorname{Tr}_{B(A)}(|\beta\rangle\langle\beta|)$ is the reduced density operator.

Lemma 2. 26] A pure bipartite state $|\beta\rangle \in \mathbb{C}^{d_{A}} \otimes \mathbb{C}^{d_{B}}\left(d_{B} \geq d_{A}\right)$ is a maximally entangled state if and only if there exits an orthonormal basis in $\mathbb{C}^{d_{B}}$ for any given orthonormal complete basis $\left\{\left|i_{A}\right\rangle\right\}$ in $\mathbb{C}^{d_{A}}$, such that $|\beta\rangle$ can be written in the following form

$$
|\beta\rangle=\frac{1}{\sqrt{d_{A}}} \sum_{i=1}^{d_{A}}\left|i_{A}\right\rangle \otimes\left|i_{B}\right\rangle .
$$

Theorem 1. An arbitrary separable state can be the output of a certain EBC corresponding exactly to the input of a maximally entangled state.

proof: Without loss of generality, suppose an arbitrary separable state $\rho=$ $\sum_{k} p_{k}\left|\psi_{k}\right\rangle\left\langle\psi_{k}|\otimes| \phi_{k}\right\rangle\left\langle\phi_{k}\right|$ in $\mathbb{C}^{d_{A}} \otimes \mathbb{C}^{d_{B}}$ and $d_{B} \geq d_{A}$. Since $\left|\psi_{k}\right\rangle$ can be represented as a linear combination of the orthomormal basis $\left\{\left|i_{A}\right\rangle\right\}$ in $\mathbb{C}^{d_{A}}$, i.e. $\left|\psi_{k}\right\rangle=\sum_{i}\left|i_{A}\right\rangle\left\langle i_{A} \mid \psi_{k}\right\rangle$, we have

$$
\begin{aligned}
\rho & \left.=\sum_{k} p_{k}\left(\sum_{i}\left|i_{A}\right\rangle\left\langle i_{A} \mid \psi_{k}\right\rangle\right)\left(\sum_{j}\left\langle j_{A}\right|\left\langle j_{A} \mid \psi_{k}\right\rangle\right)|\otimes| \phi_{k}\right\rangle\left\langle\phi_{k}\right| \\
& =\sum_{i, j, k}\left|i_{A}\right\rangle\left\langle j_{A}|\otimes| \phi_{k}\right\rangle\left\langle\phi_{k}\right| p_{k}\left\langle i_{A} \mid \psi_{k}\right\rangle\left\langle j_{A} \mid \psi_{k}\right\rangle \\
& =\sum_{i, j}\left|i_{A}\right\rangle\left\langle j_{A}\left|\otimes \sum_{k}\right| \phi_{k}\right\rangle\left\langle\phi_{k}\right| \operatorname{Tr}\left(\left|i_{A}\right\rangle\left\langle j_{A}\left|p_{k}\right| \psi_{k}\right\rangle\left\langle\psi_{k}\right|\right)
\end{aligned}
$$


The Origin of Separable States and Separability Criteria from Entanglement-breaking Channels4

$$
\begin{aligned}
& =\sum_{i, j}\left|i_{A}\right\rangle\left\langle j_{A}\left|\otimes \sum_{k}\right| \phi_{k}\right\rangle\left\langle\phi_{k}\right| \operatorname{Tr}\left(\left|i_{B}\right\rangle\left\langle j_{B}\left|p_{k}\right| \psi_{k}\right\rangle\left\langle\psi_{k}\right|\right) \\
& =\sum_{i, j}\left|i_{A}\right\rangle\left\langle j_{A}\right| \otimes \Phi\left(\left|i_{B}\right\rangle\left\langle j_{B}\right|\right) \\
& =(I \otimes \Phi)(|I\rangle\langle I|),
\end{aligned}
$$

where $\Phi(\sigma)=\sum_{k}\left|\phi_{k}\right\rangle\left\langle\phi_{k}\right| \operatorname{Tr}\left(\sigma F_{k}\right)\left(F_{k}=p_{k}\left|\psi_{k}\right\rangle\left\langle\psi_{k}\right|\right)$ is an $\mathrm{EBC},|I\rangle=\sum_{i}\left|i_{A}\right\rangle \otimes\left|i_{B}\right\rangle$ is an unnormalized maximally entangled state.

Now we give an interpretation of EBC with the input of a maximally entangled state. Since $\sum_{k} F_{k}=\sum_{k} p_{k}\left|\psi_{k}\right\rangle\left\langle\psi_{k}\right| \lesseqgtr I, \Phi(\cdot)$ is non-trace-preserving and does not provide a complete description of the processes that may occur in the system[27], the output separable state may be gotten with some probability. Let the separable state, for example, $\rho=|00\rangle\langle 00|$. With the input of the two-qubit maximally entangled state (Bell state) $\frac{1}{2}(|00\rangle\langle 00|+| 00\rangle\langle 11|+| 11\rangle\langle 00|+| 11\rangle\langle 11|)$, we have

$$
\begin{aligned}
& I \otimes \Phi\left(\frac{1}{2}(|00\rangle\langle 00|+| 00\rangle\langle 11|+| 11\rangle\langle 00|+| 11\rangle\langle 11|)\right) \\
= & |0\rangle\langle 0| \otimes\left(|0\rangle\langle 0| \operatorname{Tr}\left(\left(\frac{1}{2}(|0\rangle\langle 0|+| 0\rangle\langle 1|+| 1\rangle\langle 0|+| 1\rangle\langle 1|)\right)(|0\rangle\langle 0|)\right)\right) \\
= & \frac{1}{2}|0\rangle\langle 0|\otimes| 0\rangle\langle 0| \\
= & \frac{1}{2}|00\rangle\langle 00|,
\end{aligned}
$$

i.e. the result $|00\rangle\langle 00|$ by EBC occurs with the probability of $\frac{1}{2}$.

Rewriting Eq. (11), we have

$$
\begin{aligned}
\rho & =\frac{1}{d_{A}} \sum_{i, j}\left|i_{A}\right\rangle\left\langle j_{A}\left|\otimes d_{A} \sum_{k}\right| \phi_{k}\right\rangle\left\langle\phi_{k}\right| \operatorname{Tr}\left(\left|i_{B}\right\rangle\left\langle j_{B}\left|p_{k}\right| \psi_{k}\right\rangle\left\langle\psi_{k}\right|\right) \\
& =\frac{1}{d_{A}} \sum_{i, j}\left|i_{A}\right\rangle\left\langle j_{A}\right| \otimes \Phi^{\prime}\left(\left|i_{B}\right\rangle\left\langle j_{B}\right|\right) \\
& =\left(I \otimes \Phi^{\prime}\right)(|\beta\rangle\langle\beta|),
\end{aligned}
$$

where $\Phi^{\prime}(\sigma)=d_{A} \sum_{k}\left|\phi_{k}\right\rangle\left\langle\phi_{k}\left|\operatorname{Tr}\left(\sigma F_{k}^{\prime}\right), F_{k}^{\prime}=d_{A} p_{k}\right| \psi_{k}\right\rangle\left\langle\psi_{k}\right|$, and $|\beta\rangle=d_{A}^{-1 / 2} \sum_{i}\left|i_{A}\right\rangle \otimes\left|i_{B}\right\rangle$ is the normalized maximally entangled state. Clearly, only if one of reduced sate and all reduced sates of the separable state are maximally mixed, $\Phi^{\prime}(\cdot)$ is EBT, otherwise $\Phi^{\prime}(\cdot)$ is non-trace-preserving. The theoretical and experimental analysis of non-trace-preserving processes have been carried out [28, 29, 30].

\section{The separability criteria from entanglement-breaking channels}

By the definition of EBC, we can easily get the separability criteria from entanglementbreaking channels.

Theorem 2. A state is entangled if and only if it is not any output of the EBC.

It is impossible to travel through all EBCs, and therefore the separability criterion above is not operational. However, from the definition of EBC, we can get a class of sufficient separability criteria in matrix form. 
The Origin of Separable States and Separability Criteria from Entanglement-breaking Channels5

3.1. The separability criteria from entanglement-breaking channels with

trace-preserving

Observe the completely positive linear map $\Pi$

$$
\sigma \mapsto \Pi[\sigma]=\sum_{n=1}^{d_{B}}\left|e_{n}\right\rangle\left\langle e_{n}|\sigma| e_{n}\right\rangle\left\langle e_{n}\right|
$$

where the set $\left\{\left|e_{n}\right\rangle\right\}$ form the orthonormal basis in $\mathcal{H}_{B}$ and $\sum_{n}\left|e_{n}\right\rangle\left\langle e_{n}\right|=I$. Let $P_{n}=\left|e_{n}\right\rangle\left\langle e_{n}\right|$, we can get the following linear map

$$
\sigma \mapsto \Pi[\sigma]=\sum_{n=1}^{d_{B}} P_{n} \sigma P_{n}
$$

which is the mathematical description of the wave-packet reduction [31]. It is not hard to see that Eq.(17) is EBT.

Corollary 1. A density operator $\rho$ is separable if its entries $\rho_{i j, k l}(j \neq l)=0$, where $\rho_{i j, k l}=\langle i j|\rho| k l\rangle,\{|i\rangle\}$ and $\{|k\rangle\}$ in $\mathcal{H}_{A},\{|j\rangle\}$ and $\{|l\rangle\}$ in $\mathcal{H}_{B}$, are computational bases.

proof: An arbitrary density operator $\rho \in \mathcal{B}\left(\mathcal{H}_{A, B}\right)^{+}$can be defined as

$$
\rho=\sum_{i j k l}\langle i j|\rho| k l\rangle(|i\rangle\langle k|\otimes| j\rangle\langle l|)
$$

by computational (real orthonormal) bases $\{|i\rangle\}$ and $\{|k\rangle\}$ in $\mathcal{H}_{A},\{|j\rangle\}$ and $\{|l\rangle\}$ in $\mathcal{H}_{B}$. By Eq.(17) and Eq.(19), we have

$$
(I \otimes \Pi)[\rho]=\sum_{i j k l}\langle i j|\rho| k l\rangle\left(|i\rangle\langle k| \otimes\left(\sum_{n=1}^{d_{B}}\left|e_{n}\right\rangle\left\langle e_{n}|(|j\rangle\langle l|)| e_{n}\right\rangle\left\langle e_{n}\right|\right)\right) .
$$

Let the orthonormal basis $\left\{\left|e_{n}\right\rangle\right\}$ be the same as $\{|l\rangle\}$, it follows that

$$
(I \otimes \Pi)[\rho]=\sum_{i k l}\langle i l|\rho| k l\rangle(|i\rangle\langle k| \otimes(|l\rangle\langle l|))=\rho_{i l, k l} .
$$

This result establishes a class of the representations of matrix form for the separable state output from the EBT.

According to corollary 1, for example, a two-qubit quantum state

$$
\rho=\left(\begin{array}{cccc}
\rho_{00,00} & 0 & \rho_{00,10} & 0 \\
0 & \rho_{01,01} & 0 & \rho_{01,11} \\
\rho_{10,00} & 0 & \rho_{10,10} & 0 \\
0 & \rho_{11,01} & 0 & \rho_{11,11}
\end{array}\right)
$$

is separable.

By a simple calculation, we can get different sufficient separability criteria in matrix form with different $\left\{\left|e_{n}\right\rangle\right\}$ in Eq. (20). For example, let $\left|e_{0}\right\rangle=\frac{1}{2}(\sqrt{2}|0\rangle-|1\rangle+|2\rangle)$, $\left|e_{1}\right\rangle=\frac{1}{2}(\sqrt{2}|0\rangle+|1\rangle-|2\rangle)$ and $\left|e_{2}\right\rangle=\frac{\sqrt{2}}{2}(|1\rangle+|2\rangle)$ for qutrits, if

$$
\rho=
$$


The Origin of Separable States and Separability Criteria from Entanglement-breaking Channels6

$$
\left(\begin{array}{ccccccccc}
\rho_{00,00} & \rho_{00,01} & \rho_{00,02} & \rho_{00,10} & \rho_{00,11} & \rho_{00,12} & \rho_{00,20} & \rho_{00,21} & \rho_{00,22} \\
\rho_{01,00} & \rho_{01,01} & \rho_{01,02} & \rho_{01,10} & \rho_{01,11} & \rho_{01,12} & \rho_{01,20} & \rho_{01,21} & \rho_{01,22} \\
\rho_{02,00} & \rho_{02,01} & \rho_{02,02} & \rho_{02,10} & \rho_{02,11} & \rho_{02,02} & \rho_{02,20} & \rho_{02,21} & \rho_{02,22} \\
\rho_{10,00} & \rho_{10,01} & \rho_{10,02} & \rho_{10,10} & \rho_{10,11} & \rho_{10,12} & \rho_{10,20} & \rho_{10,21} & \rho_{10,22} \\
\rho_{11,00} & \rho_{11,01} & \rho_{11,02} & \rho_{11,10} & \rho_{11,11} & \rho_{11,12} & \rho_{11,20} & \rho_{11,21} & \rho_{11,22} \\
\rho_{12,00} & \rho_{12,01} & \rho_{12,02} & \rho_{12,10} & \rho_{12,11} & \rho_{12,02} & \rho_{12,20} & \rho_{12,21} & \rho_{12,22} \\
\rho_{20,00} & \rho_{20,01} & \rho_{20,02} & \rho_{20,10} & \rho_{20,11} & \rho_{20,12} & \rho_{20,20} & \rho_{20,21} & \rho_{20,22} \\
\rho_{21,00} & \rho_{21,01} & \rho_{21,02} & \rho_{21,10} & \rho_{21,11} & \rho_{21,12} & \rho_{21,20} & \rho_{21,21} & \rho_{21,22} \\
\rho_{22,00} & \rho_{22,01} & \rho_{22,02} & \rho_{22,10} & \rho_{22,11} & \rho_{22,02} & \rho_{22,20} & \rho_{22,21} & \rho_{22,22}
\end{array}\right)
$$

is an arbitrary density operator (entangled or not), then

$$
\rho^{\prime}=\left(\begin{array}{c|c|c}
\rho^{00} & \rho^{01} & \rho^{02} \\
\hline \rho^{10} & \rho^{11} & \rho^{12} \\
\hline \rho^{20} & \rho^{21} & \rho^{22}
\end{array}\right)
$$

is separable, where

$$
\rho^{i_{A} j_{A}}=\left(\begin{array}{ccc}
\rho_{00}^{i_{A} j_{A}} & \rho_{01}^{i_{A} j_{A}} & \rho_{02}^{i_{A} j_{A}} \\
\rho_{10}^{i_{A} j_{A}} & \rho_{11}^{i_{A} j_{A}} & \rho_{12}^{i_{A} j_{A}} \\
\rho_{20}^{i_{A} j_{A}} & \rho_{21}^{i_{A} j_{A}} & \rho_{22}^{i_{A} j_{A}}
\end{array}\right)
$$

and

$$
\begin{aligned}
\rho_{00}^{i_{A} j_{A}} & =\frac{2 \rho_{i_{A} 0, j_{A} 0}+\rho_{i_{A} 1, j_{A} 1}-\rho_{i_{A} 1, j_{A} 2}-\rho_{i_{A} 2, j_{A} 1}+\rho_{i_{A} 2, j_{A} 2}}{4}, \\
\rho_{01}^{i_{A} j_{A}} & =\frac{\rho_{i_{A} 0, j_{A} 1}-\rho_{i_{A} 0, j_{A} 2}+\rho_{i_{A} 1, j_{A} 0}-\rho_{i_{A} 2, j_{A} 0}}{4}, \\
\rho_{02}^{i_{A} j_{A}} & =\frac{-\rho_{i_{A} 0, j_{A} 1}+\rho_{i_{A} 0, j_{A} 2}-\rho_{i_{A} 1, j_{A} 0}+\rho_{i_{A} 2, j_{A} 0}}{4}, \\
\rho_{10}^{i_{A} j_{A}} & =\frac{\rho_{i_{A} 0, j_{A} 1}-\rho_{i_{A} 0, j_{A} 2}+\rho_{i_{A} 1, j_{A} 0}-\rho_{i_{A} 2, j_{A} 0}}{4}, \\
\rho_{11}^{i_{A} j_{A}} & =\frac{2 \rho_{i_{A} 0, j_{A} 0}+3 \rho_{i_{A} 1, j_{A} 1}+\rho_{i_{A} 1, j_{A} 2}+\rho_{i_{A} 2, j_{A} 1}+3 \rho_{i_{A} 2, j_{A} 2}}{8}, \\
\rho_{12}^{i_{A} j_{A}} & =\frac{-2 \rho_{i_{A} 0, j_{A} 0}+\rho_{i_{A} 1, j_{A} 1}+3 \rho_{i_{A} 1, j_{A} 2}+3 \rho_{i_{A} 2, j_{A} 1}}{8}, \\
\rho_{20}^{i_{A} j_{A}} & =\frac{-\rho_{i_{A} 0, j_{A} 1}+\rho_{i_{A} 0, j_{A} 2}-\rho_{i_{A} 1, j_{A} 0}+\rho_{i_{A} 2, j_{A} 0}}{4}, \\
\rho_{21}^{i_{A} j_{A}} & =\frac{-2 \rho_{i_{A} 0, j_{A} 0}+\rho_{i_{A} 1, j_{A} 1}+3 \rho_{i_{A} 1, j_{A} 2}+3 \rho_{i_{A} 2, j_{A} 1}}{8}, \\
\rho_{22}^{i_{A} j_{A}} & =\frac{2 \rho_{i_{A} 0, j_{A} 0}+3 \rho_{i_{A} 1, j_{A} 1}+\rho_{i_{A} 1, j_{A} 2}+\rho_{i_{A} 2, j_{A} 1}+3 \rho_{i_{A} 2, j_{A} 2}}{8}
\end{aligned}
$$

for all $0 \leq i_{A}, j_{A} \leq 2$.

Clearly, we can get different sufficient separability criteria in matrix form from different EBTs. 
The Origin of Separable States and Separability Criteria from Entanglement-breaking Channels7

3.2. The separability criteria from entanglement-breaking channels without

trace-preserving

Observe the depolarizing channel[27]

$$
D_{\epsilon}(\rho)=(1-\epsilon) \frac{I}{d_{A, B}}+\epsilon \rho .
$$

Concretely, let us consider first the case of states of two qubits. According to Ref. [14], an arbitrary density operator $\rho$ for two qubits can be written as

$$
\begin{aligned}
\rho=\frac{1}{4}[ & \left(\omega_{i} \omega_{j}+c_{i 0} \omega_{j}+\omega_{i} c_{j 0}+c_{i j}\right) P_{i} \otimes P_{j} \\
& +\left(\omega_{i} \omega_{j}-c_{i 0} \omega_{j}+\omega_{i} c_{j 0}-c_{i j}\right) \bar{P}_{i} \otimes P_{j} \\
& +\left(\omega_{i} \omega_{j}+c_{i 0} \omega_{j}-\omega_{i} c_{j 0}-c_{i j}\right) P_{i} \otimes \bar{P}_{j} \\
& \left.+\left(\omega_{i} \omega_{j}-c_{i 0} \omega_{j}-\omega_{i} c_{j 0}+c_{i j}\right) \bar{P}_{i} \otimes \bar{P}_{j}\right]
\end{aligned}
$$

and the maximally mixed density operator $I_{4}$ for two qubits may be written as

$$
\frac{1}{4}\left(\omega_{i} \omega_{j}\right)\left[P_{i} \otimes P_{j}+\bar{P}_{i} \otimes P_{j}+P_{i} \otimes \bar{P}_{j}+\bar{P}_{i} \otimes \bar{P}_{j}\right],
$$

where $I_{2}$ is the $2 \times 2$ identity matrix, $\omega_{i(j)}=1 / 3,-1 \leqslant c_{i(j) 0} \leqslant 1,-1 \leqslant c_{i j} \leqslant 1$,

$$
\begin{aligned}
& P_{i(j)}=\frac{1}{2}\left(I_{2}+\sigma_{i(j)}\right), \\
& \bar{P}_{i(j)}=\frac{1}{2}\left(I_{2}-\sigma_{i(j)}\right),
\end{aligned}
$$

$\sigma_{i(j)}$ are the Pauli matrices and $i(j)=1,2,3$.

Let

$$
\begin{aligned}
q_{0}^{i j} & =\frac{1}{4}\left(\left(\omega_{i} \omega_{j}+\epsilon\left(c_{i 0} \omega_{j}+\omega_{i} c_{j 0}+c_{i j}\right)\right)\right), \\
q_{1}^{i j} & =\frac{1}{4}\left(\left(\omega_{i} \omega_{j}+\epsilon\left(-c_{i 0} \omega_{j}+\omega_{i} c_{j 0}-c_{i j}\right)\right)\right), \\
q_{2}^{i j} & =\frac{1}{4}\left(\left(\omega_{i} \omega_{j}+\epsilon\left(c_{i 0} \omega_{j}-\omega_{i} c_{j 0}-c_{i j}\right)\right)\right), \\
q_{3}^{i j} & =\frac{1}{4}\left(\left(\omega_{i} \omega_{j}+\epsilon\left(-c_{i 0} \omega_{j}-\omega_{i} c_{j 0}+c_{i j}\right)\right)\right) .
\end{aligned}
$$

We have

$$
\begin{aligned}
D_{\epsilon}(\rho) & =(1-\epsilon) \frac{I_{4}}{d_{A, B}}+\epsilon \rho \\
& =q_{0}^{i j} P_{i} \otimes P_{j}+q_{1}^{i j} \bar{P}_{i} \otimes P_{j}+q_{2}^{i j} P_{i} \otimes \bar{P}_{j}+q_{3}^{i j} \bar{P}_{i} \otimes \bar{P}_{j} \\
& =(I \otimes \Phi)((|00\rangle+|11\rangle)(\langle 00|+\langle 11|)),
\end{aligned}
$$

where $\Phi(\sigma)=P_{j} \operatorname{Tr}\left(\sigma q_{0}^{i j} P_{i}\right)+P_{j} \operatorname{Tr}\left(\sigma q_{1}^{i j} \bar{P}_{i}\right)+\bar{P}_{j} \operatorname{Tr}\left(\sigma q_{2}^{i j} P_{i}\right)+\bar{P}_{j} \operatorname{Tr}\left(\sigma q_{3}^{i j} \bar{P}_{i}\right)$.

Clearly, $\sum_{n} q_{n}^{i j}=1$. Since $\Phi(\cdot)$ is an EBC if $q_{n}^{i j} \geqslant 0$, i.e. $\epsilon \leqslant \frac{1}{15}, D_{\epsilon}(\rho)$ is separable. Thus, the above result coincides with the result of Ref. [14].

By Eq.(35), we have

$$
D_{\epsilon}(\rho)=(1-\epsilon) \frac{I}{d_{A, B}}+\epsilon \rho
$$


The Origin of Separable States and Separability Criteria from Entanglement-breaking Channels8

$$
\begin{aligned}
& =(I \otimes \Phi)(|I\rangle\langle I|) \\
& =\sum_{i, j}\left|i_{A}\right\rangle\left\langle j_{A}\right| \otimes \Phi\left(\left|i_{B}\right\rangle\left\langle j_{B}\right|\right) \\
& =\sum_{i_{A}, j_{A}}\left|i_{A}\right\rangle\left\langle j_{A}\right| \otimes \sum_{i_{B}, j_{B}} \Phi_{i_{A}, j_{A}}\left(\left|i_{B}\right\rangle\left\langle j_{B}\right|\right)
\end{aligned}
$$

where

$$
\Phi_{i_{A}, j_{A}}\left(\left|i_{B}\right\rangle\left\langle j_{B}\right|\right)=\left\{\begin{array}{cl}
\sum_{i_{B} j_{B}}\left(\epsilon\left\langle i_{A} i_{B}|\rho| j_{A} j_{B}\right\rangle\left|i_{B}\right\rangle\left\langle j_{B}\left|+\frac{1-\epsilon}{d_{A},}\right| i_{B}\right\rangle\left\langle j_{B}\right|\right), & i=j \\
\sum_{i_{B} j_{B}}\left(\epsilon\left\langle i_{A} i_{B}|\rho| j_{A} j_{B}\right\rangle\left|i_{B}\right\rangle\left\langle j_{B}\right|\right), & i \neq j .
\end{array}\right.
$$

Since the depolarizing channel in Eq.(35) is entanglement breaking if $\epsilon \leq \frac{1}{d+1}[32$, $\Phi_{i_{A}, j_{A}}(\cdot)$ is $\mathrm{EBC}$ for all $0 \leq i_{A}, j_{A} \leq d_{A}-1$ if $\epsilon \leq \frac{1}{d+1}$.

Furthermore, for an arbitrary density operator $\rho$ (separable or not),

$$
\begin{aligned}
\rho & =(I \otimes \Psi)(|I\rangle\langle I|) \\
& =\sum_{i_{A}, j_{A}}\left|i_{A}\right\rangle\left\langle j_{A}\right| \otimes \sum_{i_{B}, j_{B}} \Psi_{i_{A}, j_{A}}\left(\left|i_{B}\right\rangle\left\langle j_{B}\right|\right)
\end{aligned}
$$

where

$$
\Psi_{i_{A}, j_{A}}\left(\left|i_{B}\right\rangle\left\langle j_{B}\right|\right)=\sum_{i_{B}, j_{B}}\left\langle i_{A} i_{B}|\rho| j_{A} j_{B}\right\rangle\left|i_{B}\right\rangle\left\langle j_{B}\right|
$$

is a map for all $0 \leq i_{A}, j_{A} \leq d_{A}-1$. By Eq.(48), we have

$$
\begin{aligned}
\Psi_{i_{A}, j_{A}}(\sigma) & =\Psi_{i_{A}, j_{A}}\left(\sum_{i_{B} j_{B}}\left\langle i_{B}|\sigma| j_{B}\right\rangle\left|i_{B}\right\rangle\left\langle j_{B}\right|\right) \\
& =\sum_{i_{B} j_{B}}\left\langle i_{A} i_{B}|\rho| j_{A} j_{B}\right\rangle\left\langle i_{B}|\sigma| j_{B}\right\rangle\left|i_{B}\right\rangle\left\langle j_{B}\right|,
\end{aligned}
$$

where $\sigma$ is any density operator in $\mathbb{C}^{d_{B}}$ and its entries $\sigma_{i_{B} j_{B}}=\left\langle i_{B}|\sigma| j_{B}\right\rangle\left|i_{B}\right\rangle\left\langle j_{B}\right|$.

An arbitrary bipartite state $\rho$ in $\mathbb{C}^{d_{A}} \otimes \mathbb{C}^{d_{B}}$ may be considered as an $d_{A} \times d_{A}$ matrix with the entries being $d_{B} \times d_{B}$ matrices.

Concretely, let

$$
\rho=\left(\begin{array}{c|c|c|c}
\rho^{00} & \rho^{01} & \ldots & \rho^{0\left(d_{A}-1\right)} \\
\hline \rho^{10} & \rho^{11} & \ldots & \rho^{1\left(d_{A}-1\right)} \\
\hline \vdots & \vdots & \ddots & \vdots \\
\hline \rho^{\left(d_{A}-1\right) 0} & \rho^{\left(d_{A}-1\right) 1} & \ldots & \rho^{\left(d_{A}-1\right)\left(d_{A}-1\right)}
\end{array}\right),
$$

where

$$
\rho^{i_{A} j_{A}}=\left(\begin{array}{cccc}
\rho_{00}^{i_{A} j_{A}} & \rho_{01}^{i_{A} j_{A}} & \ldots & \rho_{0}^{i_{A} j_{A}} \\
\rho_{10}^{i_{A} j_{A}} & \rho_{11}^{i_{A} j_{A}} & \ldots & \rho_{1\left(d_{B}-1\right)}^{\left.i_{A} j_{A}-1\right)} \\
\vdots & \vdots & \ddots & \vdots \\
\rho_{\left(d_{B}-1\right) 0} & \rho_{\left(d_{B}-1\right) 1}^{i_{A} j_{A}} & \ldots & \rho_{\left(d_{B}-1\right)\left(d_{B}-1\right)}^{i_{A} j_{A}}
\end{array}\right)
$$

for all $0 \leq i_{A}, j_{A} \leq d_{A}-1$. Therefore, we can get the following theorem.

Theorem 3. A density operator $\rho$ is separable if all blocks $\rho^{i_{A} j_{A}}\left(0 \leq i_{A}, j_{A} \leq\right.$ $\left.d_{A}-1\right)$ of $\rho$ are positive. 
proof: By Eq.(50), Eq.(51) and Eq.(52), we have

$$
\begin{aligned}
& \Psi_{i_{A}, j_{A}}(\sigma)= \\
& \left(\begin{array}{ccccc}
\rho_{00}^{i_{A} j_{A}} \cdot \sigma_{00} & \rho_{01}^{i_{A} j_{A}} \cdot \sigma_{01} & \ldots & \rho_{0\left(d_{B}-1\right)}^{i_{A} j_{A}} \cdot \sigma_{0\left(d_{B}-1\right)} \\
\rho_{10}^{i_{A} j_{A}} \cdot \sigma_{10} & \rho_{11}^{i_{A} j_{A}} \cdot \sigma_{11} & \ldots & \rho_{1\left(d_{B}-1\right)}^{i_{j_{A}}} \cdot \sigma_{1\left(d_{B}-1\right)} \\
\vdots & \vdots & \ddots & \vdots & \\
\rho_{\left(d_{B}-1\right) 0}^{i_{A} j_{A}} \cdot \sigma_{\left(d_{B}-1\right) 0} & \rho_{\left(d_{B}-1\right) 1}^{i_{A} j_{A}} \cdot \sigma_{\left(d_{B}-1\right) 1} & \ldots & \rho_{\left(d_{B}-1\right)\left(d_{B}-1\right)}^{i_{A} j_{A}} \cdot \sigma_{\left(d_{B}-1\right)\left(d_{B}-1\right)}
\end{array}\right) \\
& =\rho^{i_{A} j_{A}} \circ \sigma,
\end{aligned}
$$

where $\rho^{i_{A} j_{A}} \circ \sigma$ denotes the Hadamard product of $\rho^{i_{A} j_{A}}$ and $\sigma$. By Schur product theorem [33], since $\sigma$ is a density operator in $\mathbb{C}^{d_{B}}$ and positive, $\Psi_{i_{A}, j_{A}}(\sigma)$ is positive if $\rho^{i_{A} j_{A}}$ is positive. Since all $\rho^{i_{A} j_{A}}$ are positive, all $\Psi_{i_{A}, j_{A}}(\sigma)$ are positive. It is not hard to see that all $\Psi_{i_{A}, j_{A}}(\sigma)$ can be written in the form of Eq. (2), and therefore $\rho$ is separable.

Note that the identity map up to a common factor on subsystem does not mean the identity map on composite system for channel without trace-preserving. For example,

$$
\rho=\frac{1}{4}\left(\begin{array}{llll}
1 & 1 & 1 & 1 \\
1 & 1 & 1 & 1 \\
1 & 1 & 1 & 1 \\
1 & 1 & 1 & 1
\end{array}\right)
$$

is separable.

Clearly, the state in theorem 3 is a family of completely new separable PPT states (states which are positive under partial transposition) [16]. By Fejer's theorem[33], we have

Corollary 2. A density operator $\rho$ is separable if

$$
\sum_{i_{B}, j_{B}} \rho_{i_{B} j_{B}}^{i_{A} j_{A}} \cdot \sigma_{i_{B} j_{B}} \geqslant 0
$$

for all $0 \leq i_{A}, j_{A} \leq d_{A}-1$ and any density operator $\sigma$ in $\mathbb{C}^{d_{B}}$.

\section{Conclusion}

In conclusion, we have demonstrated that the origin of an arbitrary separable state in arbitrary composite quantum systems of arbitrary dimensions can originate from a maximally entangled state by the EBC. A class of separability criteria can be obtained from the EBC and a family of completely new separable PPT states is given. The separability criteria from EBC without trace-preserving are under investigation.

\section{Acknowledgments}

We would like to appreciate Zhihao Ma, Guang Ping He, Julio de Vicente and Mary Beth Ruskai for helpful discussions and suggestions. We would like to thank anonymous helpful comments and suggestions to improve the original manuscript. We are 
also grateful to Daowen Qiu and Guang Ping He for their excellent lectures on quantum computation and quantum information. This work is in part supported by the Key Project of NSFC-Guangdong Funds (No. U0935002).

\section{References}

[1] P. W. Shor 1994 Algorithm for quantum computation: Discrete logarithm and factoring algorithm Proceedings of the 35th Annual Symposium on Foundations of Computer Science (IEEE Computer Soceity Press, Los Alamos, CA) 124

[2] C. H. Bennett and S. J. Wiesner 1992 Communication via one- and two-particle operators on Einstein-Podolsky-Rosen states Phys. Rev. Lett. 692881

[3] C. H. Bennett, G. Brassard, C. Crepeau, R. Jozsa, A. Peres and W. K. Wootters 1993 Teleporting an unknown quantum state via dual classical and Einstein-Podolsky-Rosen channels Phys. Rev. Lett. 701895

[4] A. K. Ekert 1991 Quantum cryptography based on Bells theorem Phys. Rev. Lett. 67661

[5] R. Horodecki, P. Horodecki, M. Horodecki and K. Horodecki 2009 Quantum entanglement Rev. Mod. Phys. 81865

[6] A. Peres 1996 Separability Criterion for Density Matrices Phys. Rev. Lett. 771413

[7] R. Horodecki, P. Horodecki and M. Horodecki 1996 Quantum $\alpha$-entropy inequalities: independent condition for local realism? Phys. Lett. A 210377

[8] P. Horodecki 1997 Separability criterion and inseparable mixed states with positive partial transposition Phys. Lett. A 232333

[9] M. Horodecki and P. Horodecki 1999 Reduction criterion of separability and limits for a class of distillation protocols Phys. Rev. A 594206

[10] M. A. Nielsen and J. Kempe 2001 Separable States Are More Disordered Globally than Locally Phys. Rev. Lett. 865184

[11] O. Rudolph 2003 Some properties of the computable cross-norm criterion for separability Phys. Rev. A 67032312

[12] K. Chen and L. A. Wu 2003 A matrix realignment method for recognizing entanglement Quant. Inf. Comp. 3193

[13] O. Gühne, P. Hyllus, O. Gittsovich and J. Eisert 2007 Covariance Matrices and the Separability Problem Phys. Rev. Lett. 99130504

[14] S. L. Braunstein, C. M. Caves, R. Jozsa, N. Linden, S. Popescu and R. Schack 1999 Separability of Very Noisy Mixed States and Implications for NMR Quantum Computing Phys. Rev. Lett. 831054

[15] A. O. Pittenger and M. H. Rubin 2000 Separability and Fourier representations of density matrices Phys. Rev. A 62032313

[16] M. Horodecki, P. Horodecki and R. Horodecki 1996 Separability of mixed states: necessary and sufficient conditions Phys. Lett. A 2231

[17] E. Schrödinger 1935 Die gegenwartige Situation in der Quantenmechanik (The Current Situation in Quantum Mechanics) Naturwissenschaften 23807.

[18] K. Kraus 1983 States, Effects and Operations: Fundamental Notions of Quantum Theory (Springer-Verlag)

[19] F. Verstraete and H. Verschelde 2003 On quantum channels arXiv:quant-ph/0202124v2

[20] A. S. Holevo 1999 Coding theorems for quantum channels Russian Math. Surveys $\mathbf{5 3} 1295$

[21] M. Horodecki, P. W. Shor and M. B. Ruskai 2003 Entanglement breaking channels Rev. Math. Phys. 15629

[22] M. B. Ruskai 2003 Qubit entanglement breaking channels Rev. Math. Phys. 15643 
[23] P. W. Shor 2004 Equivalence of additivity questions in quantum information theory Commun. Math. Phys. 246453

[24] J. K. Korbicz, M. L. Almeida, J. Bae, M. Lewenstein and A. Acin 2008 Structural approximations to positive maps and entanglement-breaking channels Phys. Rev. A 78062105

[25] M. F. Sacchi 2005 Entanglement can enhance the distinguishability of entanglement-breaking channels Phys. Rev. A $\mathbf{7 2} 014305$

[26] Zong-Guo Li, Ming-Jing Zhao, Shao-Ming Fei, Heng Fan and W. M. Liu 2009 Maximally entangled state can be a mixed state arXiv:0906.5445

[27] M. A. Nielsen and I.L. Chuang 2000 Quantum Computation and Quantum Information (Cambridge Univ. Press, Cambridge)

[28] N. Kiesel, C. Schmid, U. Weber, G.Tth, O. Gühne, R. Ursin and H. Weinfurter 2005 Experimental Analysis of a Four-Qubit Photon Cluster State Phys. Rev. Lett. 95210502

[29] A. G. Kofman and A. N. Korotkov 2009 Two-qubit decoherence mechanisms revealed via quantum process tomography Phys. Rev. A 80042103

[30] I. Bongioanni, L. Sansoni, F. Sciarrino, G. Vallone and P. Mataloni 2010 Experimental quantum process tomography of non trace-preserving maps arXiv:1008.5334

[31] F. Benatti and R. Floreanini 2005 Open quantum dynamics: complete positivity and entanglement Int. J. Mod. Phys. B 19, 3063

[32] A. Grudka and P. Horodecki 2010 Nonadditivity of quantum and classical capacities for entanglement breaking multiple-access channels and butterfly network Phys. Rev. A $\mathbf{8 1}$ 060305(R)

[33] R. A. Horn and C. R. Johnson 1990 Matrix Analysis (Cambridge Univ. Press, Cambridge) 